PROCEEDINGS OF THE

AMERICAN MATHEMATICAL SOCIETY

Volume 127, Number 10, Pages 3027-3034

S 0002-9939(99)04889-3

Article electronically published on April 23, 1999

\title{
A NOTE ON RIGIDITY OF 3-SASAKIAN MANIFOLDS
}

\author{
HENRIK PEDERSEN AND YAT SUN POON
}

(Communicated by Peter Li)

\begin{abstract}
Making use of the relations among 3-Sasakian manifolds, hypercomplex manifolds and quaternionic Kähler orbifolds, we prove that complete 3-Sasakian manifolds are rigid.
\end{abstract}

\section{INTRODUCTION}

Recent investigations of 3-Sasakian structures are tied to the investigations of quaternionic Kähler manifolds [5], [6]. Boyer, Galicki and Mann find that over any quaternionic Kähler manifold, there is a principal fiber bundle with group $\mathrm{SO}(3)$ such that the total space is a 3-Sasakian manifold. More important, they find that every complete 3 -Sasakian manifold $\mathcal{S}$ fibers over a quaternionic Kähler orbifold $M$ [5]. Using a reduction process with roots in symplectic reduction and hyper-Kähler reduction [10], Boyer et al. produce a large discrete family of 3-Sasakian structures [6]. The need for studying deformations of 3-Sasakian structures starts to emerge after their construction. It is further discovered that the product space $\mathbf{R}^{+} \times \mathcal{S}$ is a hyper-Kähler manifold [6]. It is isomorphic to the associated bundle of $M$ as defined by Swann [19]. When $\mathcal{S}$ is compact, the discrete quotient $X=S^{1} \times \mathcal{S}=$ $\left(\mathbf{R}^{+} / \mathbf{Z}\right) \times \mathcal{S}$ is a compact hyper-Hermitian manifold. The relations among the 3 Sasakian manifold $\mathcal{S}$, the hyper-Hermitian manifold $X$ and the quaternionic Kähler orbifold $M$ allow us to apply the theory of deformations of complex manifolds [16] to study the deformations of 3-Sasakian structures. In this note, we shall prove the following theorem.

Theorem. Complete 3-Sasakian manifolds are rigid.

LeBrun shows that compact quaternionic Kähler manifolds with positive scalar curvature are rigid [13]. Due to the correspondence between compact 3-Sasakian manifolds and compact quaternionic Kähler orbifolds, the above theorem, in the context of quaternionic Kähler geometry, is a generalization of LeBrun's result.

\section{RIGIDITY OF COMPLETE 3-SASAKIAN MANIFOLDS}

A $4 n+3$-dimensional Riemannian manifold $(\mathcal{S}, g)$ has a Sasakian structure if there is a unit Killing vector field $V$ such that for any pair of vector fields $A$ and $B$

Received by the editors December 4, 1997.

1991 Mathematics Subject Classification. Primary 53C25; Secondary 32G05, 53 C55.

Key words and phrases. Sasakian, hypercomplex, deformations.

The first author was partially supported by NATO CRG-950040. The second author was partially supported by NSF grant DMS-9504908.

(c)1999 American Mathematical Society 
on $\mathcal{S},\left(\nabla_{A} \nabla V\right) B=g(B, V) A-g(A, B) V[20]$. The manifold $(\mathcal{S}, g)$ has a 3-Sasakian structure if there are three unit Killing vector fields $\left\{V_{1}, V_{2}, V_{3}\right\}$ such that each of these vector fields determines a Sasakian structure, and the algebra generated by these vector fields is isomorphic to $\mathfrak{s u}(2)$. A 3-Sasakian manifold is complete if the vector fields $\left\{V_{1}, V_{2}, V_{3}\right\}$ are complete [6]. In such a case the group $G=\mathrm{SO}(3)$ or $\mathrm{SU}(2)$ acts on $\mathcal{S}$ as a group of isometries. We sacrifice the requirement that the group $G$ acts on $\mathcal{S}$ effectively to the simplicity of presentation. We assume that $G$ is the group $\mathrm{SU}(2)$.

By [6, Theorem A], a complete 3-Sasakian manifold $\mathcal{S}$ is a compact Einstein manifold with positive scalar curvature. The space of leaves $M$ of the $G$-action on $\mathcal{S}$ is a quaternionic Kähler orbifold of dimension $4 n$ with positive scalar curvature. By [6, Theorem B], the cone metric $d r^{2}+r^{2} g$ on the product manifold $\mathcal{U}=\mathbf{R}^{+} \times \mathcal{S}$ is hyper-Kähler. Furthermore, the space $\mathcal{U}$ is the associated bundle of the orbifold $M$ in the sense of Swann [19]. When $\langle\lambda\rangle$ is the multiplicative group generated by a real number $\lambda$ between 0 and 1 , let $X$ be the quotient space $S^{1} \times \mathcal{S}=\left(\mathbf{R}^{+} /\langle\lambda\rangle\right) \times \mathcal{S}$. With the induced hypercomplex structure and the product metric $\frac{d r^{2}}{r^{2}}+g, X$ is a hyper-Hermitian manifold.

Let $Z$ and $W$ be the twistor spaces of $M$ and $X$ respectively. Let $F$ be the space $S^{1} \times G=\mathbf{H}^{*} /\langle\lambda\rangle$. For any finite subgroup $\Gamma$ of $G$, define $F_{\Gamma}=S^{1} \times(G / \Gamma)$. As $G$ is a group of quaternionic transformations on $\mathcal{U}$, and $\mathbf{R}^{+}$is a group of hypercomplex transformations [6], [19], $S^{1} \times G$ is lifted to a group of holomorphic transformations on the twistor space of $X$ [16]. As the orbits of this group on $X$ is invariant of the hypercomplex structure [6], the induced foliations on the twistor spaces are holomorphic. Therefore, we have

Lemma 1. There exists a holomorphic foliation $\mathcal{F}$ on the twistor space $W$ of the hypercomplex manifold $X$ such that its leaves are $F_{\Gamma}$ for some $\Gamma$.

Lemma 2. The space of leaves of the foliation $\mathcal{F}$ on $W$ is isomorphic, as a holomorphic orbifold, to the twistor space $Z$ of the quaternionic Kähler orbifold $M$.

Proof. Since the leaves of the foliation are compact, $W / \mathcal{F}$ is a complex orbifold [15]. The singularities of this orbifold are images of leaves with non-trivial leave holonomy [15]. Let $\Gamma$ be the isotropy subgroup of the action $U(1) \times G$ at a point $w$ on a leave with non-trivial holonomy. Let $\pi$ be the twistor projection from $W$ onto $X=S^{1} \times \mathcal{S}$. When $\pi(w)=(t, s)$, then for any element $\phi$ in $G, \pi(\phi(w))=(t, \phi(s))$. Therefore, if $\Gamma$ is an isotropic subgroup of the $G$-action on $W$ at the point $w$, then $\Gamma$ is contained in the isotropic subgroup of the $G$-action on $\mathcal{S}$ at the point $s$. Conversely, when $\Gamma$ is the isotropic subgroup of the $G$-action at a point $s$ on $\mathcal{S}$, it is the isotropic subgroup of the $G$-action at $(t, s)$ on the space $X$. Since $X$ is hypercomplex and the $G$-action on $X$ preserves the underlying quaternionic structure, the range of the isotropy representation of $\Gamma$ is contained in $\operatorname{GL}(n+1, \mathbf{H}) \mathrm{Sp}(1)$. The twistor space $W$ is an associated fiber bundle on $X$ defined by the standard representation $[18]$

$$
\mathrm{GL}(n+1, \mathbf{H}) \mathrm{Sp}(1) \rightarrow \mathrm{SO}(3)
$$

and the natural action of $\mathrm{SO}(3)$ on the 2 -sphere. Composing these representations with the isotropy representation, we obtain the induced action of $\Gamma$ on the twistor line over $(t, s)$. As $\Gamma$ is Abelian [4], the induced action of $\Gamma$ on the twistor line over $(t, s)$ has a fixed point $w$, and $\Gamma$ is contained in the isotropy subgroup of the $G$ action on the twistor space $W$. Hence, there is a one-to-one correspondence between 
the singularities of $Z$ and singular orbits of the $U(1) \times G$-action on $W$. By taking quotient with respect to the $U(1) \times G$-action, we have a homeomorphism between the topological spaces $W / \mathcal{F}$ and $Z$. By [16, Lemma 4.1], this homeomorphism is an analytic isomorphism outside the singular sets of these two complex orbifolds. By analytic continuation, the homeomorphism is an analytic isomorphism over the entire space.

When $\left(\mathcal{S}_{t}, g_{t}, G_{t}\right)$ is a deformation of the 3-Sasakian structure $(\mathcal{S}, g, G), X_{t}=$ $S^{1} \times \mathcal{S}_{t}$ is a deformation of the compact hyper-Hermitian space $X=S^{1} \times \mathcal{S}$, with the product metric $g_{X_{t}}=\frac{d r^{2}+r^{2} g_{t}}{r^{2}}=(d \ln r)^{2}+g_{t}$.

To study deformations of the hypercomplex structure on $X$, we consider deformations of the associated twistor space $W$. Due to the twistor correspondence [18], [16], the deformation theory of the underlying quaternionic structure on $X$ is described by the cohomology groups of $\Theta_{W}$. Since $X$ is hypercomplex, there is a holomorphic projection $p$ from $W$ to $\mathbf{C P}^{1}$ such that each fiber is the underlying differentiable structure on $X$ with a choice of complex structure [10], [16]. On the other hand, the fibers of the natural projection $\Phi$ from $W$ to the leave space $Z$ of the action of $U(1) \times G$ is an elliptic Hopf surface. The intersection of the fibers of the maps $p$ and $\Phi$ is the orbit of the holomorphic action of the subgroup $U(1) \times U(1)$ in $U(1) \times G$. Therefore, the fiber of the product map $\Phi \times p$ is an elliptic curve. The kernel of the differential of the product map is a trivial bundle. It defines the following exact sequence on $W$ :

$$
0 \rightarrow \mathcal{O}_{W} \rightarrow \Theta_{W} \rightarrow \Phi^{*} \Theta_{Z} \oplus p^{*} \Theta_{\mathbf{C P}^{1}} \rightarrow 0
$$

We use the induced long exact sequence of this sequence to calculate the cohomology of the tangent sheaf $\Theta_{W}$. To calculate cohomology groups, we want to apply spectral sequences to compute the direct image sheaves via the maps $p$ and $\Phi$ as in [16, Section 5]. Since the twistor space $Z$ is an orbifold, we need the following technical observations so that we can adopt the computations in [16].

Lemma 3. On a complex orbifold $Z$, let $\Theta$ be its tangent $V$-bundle, and $\Omega$ be the sheaf of Kähler differential. Then $\operatorname{Ext}^{n}(Z ; \Omega, \mathcal{O})=H^{n}(Z, \Theta)$ for all $n \geq 0$.

Proof. On a local uniformization neighborhood $U / \Gamma$ on $Z[2]$,

$$
\mathcal{H o m}(\Omega, \mathcal{O})(U / \Gamma)=\mathcal{H o m}(\Omega, \mathcal{O})_{\Gamma}(U)=\Theta(U)_{\Gamma}=\Theta(U / \Gamma) .
$$

Therefore we have an isomorphism $\mathcal{H o m}(\Omega, \mathcal{O}) \cong \Theta_{Z}$. For any projective resolution of $\Omega$

$$
\cdots \rightarrow E_{m}(U / \Gamma) \rightarrow E_{m-1}(U / \Gamma) \rightarrow \cdots \rightarrow E_{0}(U / \Gamma) \rightarrow \Omega(U / \Gamma) \rightarrow 0
$$

the sheaf $\mathcal{E} x t^{p}(Z ; \Omega, \mathcal{O})$ over $(U / \Gamma)$ is the $p$-th cohomology of the induced resolution. When the induced resolution is pulled back to a resolution on $U$, we have a complex of $\Gamma$-invariant spaces:

$$
0 \rightarrow \mathcal{H o m}(\Omega(U), \mathcal{O})_{\Gamma} \rightarrow \cdots \rightarrow \mathcal{H o m}\left(E_{m}(U), \mathcal{O}\right)_{\Gamma} \rightarrow \cdots
$$

As $U$ is non-singular and $\Omega(U)$ is locally free, when $U$ is small, $\mathcal{H o m}\left(E_{m}(U), \mathcal{O}\right)_{\Gamma}=$ 0 for all $m \geq 1$. Therefore, $\mathcal{E} t^{p}(Z ; \Omega, \mathcal{O})=0$ for all $p \geq 1$, and $\mathcal{H o m}(\Omega, \mathcal{O}) \cong$ $\mathcal{E} x t^{0}(Z ; \Omega, \mathcal{O})$.

There is a local-to-global spectral sequence such that $E_{2}^{p, q}=H^{p}\left(Z, \mathcal{E} x t^{q}(\Omega ; \mathcal{O})\right)$, and $E_{\infty}^{p . q}=E x t^{p+q}(Z ; \Omega, \mathcal{O})[8],[9]$. The conclusion of the previous paragraph implies that this spectral sequence degenerates at $E_{2}$-level. It yields the isomorphism $\operatorname{Ext}^{n}(Z ; \Omega, \mathcal{O})=H^{n}\left(Z, \Theta_{Z}\right)$ for all $n \geq 0$. 
Lemma 4. When $\Phi: W \rightarrow Z$ is the quotient map defined by the leaves of the foliation $\mathcal{F}$, and $\mathcal{L}$ is the sheaf of germs of sections of a holomorphic $V$-bundle on $Z$, then $R^{j} \Phi_{*}\left(\Phi^{*} \mathcal{L}\right)=\left(R^{j} \Phi_{*} \mathcal{O}_{W}\right) \otimes \mathcal{L}$.

Proof. The proof of this lemma is similar to the above one. Let $U / \Gamma$ be a local uniformization chart on $Z$ so that $U$ is a slice of the foliation. Let $q$ be the quotient map $U \rightarrow U / \Gamma$, and $\Psi$ a map from $\Phi^{-1}(U / \Gamma)$ to $U$ so that $\Phi=q \circ \Psi$. Then

$$
R^{j} \Phi_{*}\left(\Phi^{*} \mathcal{L}\right)(U / \Gamma)=H^{j}\left(\Phi^{-1}(U / \Gamma), \Phi^{*} \mathcal{L}\right)=H^{j}\left(\Psi^{-1}(U), \Psi^{*} q^{*} \mathcal{L}\right)_{\Gamma}
$$

When $U$ is so small that $\left(q^{*} \mathcal{L}\right)(U)$ is a free, then

$H^{j}\left(\Psi^{-1}(U), \Psi^{*} q^{*} \mathcal{L}\right)_{\Gamma}=\left(H^{j}\left(\Psi^{-1}(U), \mathcal{O}_{W}\right) \otimes\left(q^{*} \mathcal{L}\right)(U)\right)_{\Gamma}=\left(R^{j} \Phi_{*} \mathcal{O}_{W} \otimes \mathcal{L}\right)(U / \Gamma)$.

When the twistor space $Z$ is smooth, the map $\Phi$ is a locally trivial fibration. Then the computation of the direct image of the structure sheaf of $W$ is easy. When the twistor space is an orbifold, the computation is not hard.

Lemma 5. For $j \geq 2, R^{j} \Phi_{*} \mathcal{O}_{W}=0$. Moreover, $R^{0} \Phi_{*} \mathcal{O}_{W}=\mathcal{O}_{Z} \otimes H^{0}\left(F, \mathcal{O}_{F}\right) \cong$ $\mathcal{O}_{Z} ; R^{1} \Phi_{*} \mathcal{O}_{W}=\mathcal{O}_{Z} \otimes H^{1}\left(F, \mathcal{O}_{F}\right) \cong \mathcal{O}_{Z}$.

Proof. We continue to use the notation of the last two lemmata. By Kunneth's formula and the $\bar{\partial}$-Poincare Lemma,

$$
R^{j} \Phi_{*} \mathcal{O}_{W}=H^{j}\left(\Psi^{-1}(U), \mathcal{O}_{W}\right)_{\Gamma}=\left(H^{0}\left(U, \mathcal{O}_{U}\right) \otimes H^{j}\left(F, \mathcal{O}_{F}\right)\right)_{\Gamma}
$$

The first statement of this lemma follows from the vanishing of $H^{j}\left(F, \mathcal{O}_{F}\right)$ for $j \geq 2$. When $j$ is either 0 or $1, H^{j}\left(F, \mathcal{O}_{F}\right)_{\Gamma}=H^{j}\left(F, \mathcal{O}_{F}\right)$. It is because when one considers $F$ as a homogeneous complex manifold $\mathrm{SO}(2) \times \mathrm{SU}(2), H^{j}\left(F, \Theta_{F}\right)$ is identified to the adjoint representation space $(\mathfrak{s o}(2) \oplus \mathfrak{s u}(2))_{\mathbf{C}}$ when $j=0,1$. The left action of $\mathrm{SO}(2)$ generates a nowhere vanishing holomorphic vector field. This vector field defines a bundle map from the structure sheaf into the tangent sheaf. Through this map, $H^{j}\left(F, \mathcal{O}_{F}\right)$ is identified to the $\mathfrak{s o}(2)_{\mathbf{C}}$ summand in $H^{j}\left(F, \Theta_{F}\right)$. It follows that the action of $\Gamma$ on $H^{j}\left(F, \mathcal{O}_{F}\right)$ is trivial, because $\Gamma$ is a subgroup of $\mathrm{SU}(2)$. By (2), $R^{j} \Phi_{*} \mathcal{O}_{W}=H^{0}\left(U, \mathcal{O}_{U}\right)_{\Gamma} \otimes H^{j}\left(F, \mathcal{O}_{F}\right)$. The claims in the lemma follow.

Lemma 6. Let $Z$ be the twistor space of a complete 3-Sasakian manifold $\mathcal{S}$. Then (a) $H^{p}(Z, \mathcal{O})=\{0\}$ for all $p \geq 1$; and (b) $H^{p}\left(Z, \Theta_{Z}\right)=\{0\}$ for all $p \geq 2$.

Proof. The twistor space $Z$ has a Kähler-Einstein orbifold metric with positive scalar curvature [4], [17]. If the twistor space is smooth, the two vanishing theorems in this lemma are consequences of the classical Kodaira Vanishing Theorem and Akizuki-Nakano Vanishing Theorem [1], [12]. To prove these two vanishing theorems, one begins with establishing the Dolbeault's harmonic theory on Kähler manifolds and the Serre duality. Using harmonic representatives for elements in cohomology classes, one proves these vanishing theorems by a Bochner type argument.

Noting that an orbifold is locally finitely covered by smooth manifolds. Baily defined $L^{2}$-norms on Riemannian orbifolds by an averaging process with respect to the local covers of each orbifold point [2, page 866]. Thereafter, he established the harmonic theory. Using the same averaging process, he proved the Dolbeault Theorem [3, page 405], and completed the proof of the Kodaira Vanishing Theorem by a Bochner type argument [3, page 411]. Therefore, Part (a) follows directly from Baily's works. 
The Dolbeault Theorem for a $V$-bundle on orbifolds remains valid. Using the $L^{2}$-norm defined by Baily, one can complete the proof of Part (b) by producing the Nakano Inequality as presented in [12, page 69]. Then Kobayashi's brief discussion after the Nakano Inequality [12, page 69] is applied to verify Part (b).

Using the projection formula given by Lemma 4, the computation in Lemma 5 and the above vanishing theorems, we can apply various lemmata in [16, Section 5] even when $Z$ is an orbifold. The conclusion is that the induced long exact sequence of (1) splits on each cohomology level. Furthermore, the cohomology of the pullback bundles on $W$ are isomorphic to the cohomology of the pull-back bundles on product spaces. Therefore, the virtual parameter space for the deformations of the complex structure on $W$ is given as in [16, (5.8)]:

$$
\begin{aligned}
H^{1}\left(W, \Theta_{W}\right)= & H^{0}\left(Z, \mathcal{O}_{Z}\right) \otimes H^{1}\left(F, \mathcal{O}_{F}\right) \oplus H^{0}\left(Z, \Theta_{Z}\right) \otimes H^{1}\left(F, \mathcal{O}_{F}\right) \\
& \oplus H^{1}\left(Z, \Theta_{Z}\right) \otimes H^{0}\left(F, \mathcal{O}_{F}\right) \oplus H^{1}\left(W, p^{*} \Theta_{\mathbf{C P}^{1}}\right) .
\end{aligned}
$$

To take metric geometry into consideration, we recall that the orbifold twistor space $Z$ is a contact space. Let $\mathcal{E}$ be the kernel of the contact 1 -form $\theta$. It determines the following exact sequence of $V$-bundles on the orbifold $Z$ :

$$
0 \rightarrow \mathcal{E} \rightarrow \Theta_{Z} \stackrel{\theta}{\rightarrow} K^{-1 / n+1} \rightarrow 0,
$$

where $K$ is the canonical bundle on $Z$. When the twistor space $Z$ is smooth, LeBrun finds [14] that for all $j$, there is a splitting of cohomology groups $H^{j}\left(Z, \Theta_{Z}\right)=$ $H^{j}(Z, \mathcal{E}) \oplus H^{j}\left(Z, K^{-1 / n+1}\right)$. With the aid of Lemma 4,5 and 6 , a spectral sequence argument shows that

$$
\begin{aligned}
H^{1}\left(W, \Phi^{*} K^{-1 / n+1}\right) & =H^{0}\left(Z, K^{-1 / n+1}\right) \otimes H^{1}\left(F, \mathcal{O}_{F}\right) \\
& \oplus H^{1}\left(Z, K^{-1 / n+1}\right) \otimes H^{0}\left(F, \mathcal{O}_{F}\right) .
\end{aligned}
$$

Moreover, the infinitesimal deformations of a contact structure on $Z$ are described by Cech 1-cocyles with coefficients in the bundle $K^{-1 / n+1}$ [13]. Therefore, if $\alpha$ is an infinitesimal deformation of $W$ arising from infinitesimal deformations of 3-Sasakian structures, then $d \Phi(\alpha)$ is contained in $H^{1}\left(W, \Phi^{*} K^{-1 / n+1}\right)$. This computation remains valid when $Z$ is an orbifold. Hence, if an infinitesimal deformation $\alpha$ arises from infinitesimal deformations of the 3-Sasakian structure on $\mathcal{S}$, it is contained in a subspace of $H^{1}\left(W, \Theta_{W}\right)$. In particular, the second and third summands in (3) are now replaced by $H^{1}\left(W, \Phi^{*} K^{-1 / n+1}\right)$. By (5), we find that $\alpha$ is contained in

$$
\begin{gathered}
H^{0}\left(Z, \mathcal{O}_{Z}\right) \otimes H^{1}\left(F, \mathcal{O}_{F}\right) \oplus H^{0}\left(Z, K^{-1 / n+1}\right) \otimes H^{1}\left(F, \mathcal{O}_{F}\right) \\
\oplus H^{1}\left(Z, K^{-1 / n+1}\right) \otimes H^{0}\left(F, \mathcal{O}_{F}\right) \oplus H^{1}\left(W, p^{*} \Theta_{\mathbf{C P}^{1}}\right) .
\end{gathered}
$$

To complete the proof of the main theorem, we claim that no element in this space is induced by any deformations of the 3 -Sasakian structure on $\mathcal{S}$. Let us analyze its four summands, starting from the last one.

Any non-trivial deformation of the 3-Sasakian structure on $S$ induces a nontrivial deformation of the hypercomplex structure on $X$. By a twistor correspondence, it induces a non-trivial deformation of the map $p$. Due to Horikawa [11], deformation theory of the map $p$ is described in the cohomology groups of the kernel of the differential $d p$. Therefore, the summand $H^{1}\left(W, p^{*} \Theta_{\mathbf{C P}^{1}}\right)$ does not contribute to non-trivial deformations of the hypercomplex structure on $X$. 
Since the orbifold $Z$ is Kähler-Einstein with positive scalar curvature [4], [17], due to the Kodaira-Baily Vanishing Theorem [3], [4], $H^{1}\left(Z, K^{-1 / n+1}\right)$ vanishes. Therefore, the third summand in (6) vanishes.

Recall that the group $G$ of isometries on $\mathcal{S}$ acts on $X=S^{1} \times \mathcal{S}$ as a group of quaternionic transformations, and the rotation group $U(1)$ of the $S^{1}$-factor is a group of hypercomplex transformations [6]. By a twistor correspondence [16], the product $U(1) \times G$ is a group of holomorphic transformations on the twistor space $W$. As these observations are applied to any complete 3-Sasakian manifold, the deformations of $W$ induced from deformations of the 3-Sasakian structure on $\mathcal{S}$ are $U(1) \times G$-equivariant. By Cathelineau's theory [7], equivariant deformations are parameterized by invariant elements of the virtual parameter space. We claim that the second summand in (6) does not contain invariant elements. When a complex structure on $X$ is chosen, it can be recognized as follows. Let $L^{*}$ be the principal $\mathbf{C}^{*}$-bundle of $K^{1 /(n+1)}$ over $Z$. Then $X$ is the discrete quotient of $L^{*}$ by the integer subgroup of $\mathbf{C}^{*}$ generated by the multiplication of a real number $\lambda$ in the open interval $(0,1)$. The group $U(1) \times G$ contains a subgroup $U(1) \times U(1)$ which is the quotient of the principal group $\mathbf{C}^{*}$ by $\langle\lambda\rangle$. Therefore, the action of $U(1) \times U(1)$ on $X$ is through the fiberwise multiplication of $\mathbf{C}^{*}$ on $L^{*}$. Its induced action on $K^{-1 / n+1}$ is the fiberwise inverse multiplication of $\mathbf{C}^{*}$. It follows that no section of the bundle $K^{-1 / n+1}$ is invariant of the group $U(1) \times U(1)$. We claim that the $U(1) \times G$-action on the factor $H^{1}\left(F, \mathcal{O}_{F}\right)$ in the second summand of (6) is trivial. This claim together with the above observation on $H^{0}\left(Z, K^{-1 / n+1}\right)$ implies that the second summand does not contain invariant elements. Recall that $F$ is a homogeneous complex manifold with respect to the group $U(1) \times G$. The cohomology $H^{1}\left(F, \Theta_{F}\right)$ is identified to the adjoint representation of the complexified Lie algebra $(\mathfrak{u}(1) \oplus \mathfrak{g})_{\mathbf{C}}$. The nowhere vanishing holomorphic vector field generated by $U(1)$ defines the cohomology $H^{1}\left(F, \mathcal{O}_{F}\right)$ as a subspace of $H^{1}\left(F, \Theta_{F}\right)$. As a subalgebra, it is precisely $\mathfrak{u}(1)_{\mathbf{C}}$. Therefore, the action of $U(1) \times G$ on $H^{1}\left(F, \mathcal{O}_{F}\right)$ is trivial.

To examine the first summand, note that when $\left(\mathcal{S}_{t}, g_{t}, G_{t}\right)$ is a deformation of $(\mathcal{S}, g, G), \mathcal{U}_{t}=\mathbf{R}^{+} \times \mathcal{S}_{t}$ is a deformation of $\mathcal{U}$. When $\lambda$ is a real number between 0 and 1 , we construct the quotient space $X_{t}(\lambda)=\mathbf{R}^{+} /\langle\lambda\rangle \times \mathcal{S}_{t}$ with the product metric $g_{(t, \lambda)}=(d \ln r)^{2}+g_{t}$. Since the length of the circle $\mathbf{R}^{+} /\langle\lambda\rangle$ with respect to $g_{(t, \lambda)}$ is a non-trivial function of $\lambda, \lambda$ is a non-trivial parameter for the deformation of hyper-Hermitian structures of $X$. However, the leave space of the $U(1)$-action on $X_{t}(\lambda)$ for various $\lambda$ defines one 3-Sasakian structure $\mathcal{S}_{t}$. Therefore, the length of the holomorphic vector field generated by the $U(1)$ action is a redundant parameter for the deformations of the 3-Sasakian structures on $\mathcal{S}$. As this parameter spans the first summand [16], the first summand does not parameterize non-trivial deformations of the 3-Sasakian structure on $\mathcal{S}$.

Since no element in the space given in (6) represents infinitesimal deformations of any non-trivial deformations of 3-Sasakian structures, complete 3-Sasakian structures are rigid as claimed.

Remark. The quotient of a 3-Sasakian manifold $\mathcal{S}$ by a circle subgroup in $\mathrm{SU}(2)$ is the twistor space $Z$ of the quaternionic Kähler orbifold $M$ [4]. Based on LeBrun's work, we observed in the last few paragraphs that the contact structure on $Z$ is rigid. To prove the main theorem given in the introduction, it suffices to prove that the quotient map from $\mathcal{S}$ to $Z$ is smoothly rigid. By considering the twistor 
space $W$ of the hypercomplex manifold $\mathcal{S} \times S^{1}$, we proved that the holomorphic map from $W$ to $Z$ is complex analytically and equivariantly rigid. Therefore, our approach to verify the rigidity of compact 3-Sasakian manifolds may not be the most effective one. However, our computation contains information relevant to quaternionic geometry. The isomorphism (3) provides a virtual parameter count for the deformations of quaternionic structures on the hypercomplex manifold $\mathcal{S} \times S^{1}$. The virtual parameter count for the deformations of hypercomplex structures, as explained in [16], is given by

(7)

$$
H^{0}\left(Z, \mathcal{O}_{Z}\right) \otimes H^{1}\left(F, \mathcal{O}_{F}\right) \oplus H^{0}\left(Z, \Theta_{Z}\right) \otimes H^{1}\left(F, \mathcal{O}_{F}\right) \oplus H^{1}\left(Z, \Theta_{Z}\right) \otimes H^{0}\left(F, \mathcal{O}_{F}\right) .
$$

Therefore, we have improved some results in [16] by including orbifolds in our considerations here.

\section{ACKNOWLEDGMENT}

Our result on the rigidity of 3-Sasakian structures was anticipated by C. Boyer before we worked out a proof in detail. We thank him for useful conversations and encouragement during the course of our work. We also thank the referee for the useful comments.

\section{REFERENCES}

[1] Y. Akizuki \& S. Nakano. Note on Kodaira-Spencer's proof of Lefschetz theorems, Proc. Japan Acad., 30 (1954) 266-272. MR 16:619a

[2] W. L. Baily. The decomposition theorem for V-manifolds, Amer. J. Math., 78 (1956) 862-888. MR 20:6537

[3] W. L. Baily. On the imbedding of V-manifolds in projective space, Amer. J. Math., 79 (1957) 403-430. MR 20:6538

[4] C. Boyer \& K. Galicki. The twistor space of a 3-Sasakian manifold, International J. Math., 8 (1997) 31-60. MR 98e:53072

[5] C. Boyer, K. Galicki \& B. Mann. Quaternionic reduction and Einstein manifolds, Comm. Anal. Geom., 1 (1993) 229-279. MR 95c:53026

[6] C. Boyer, K. Galicki \& B. Mann. The geometry and topology of 3-Sasakian manifolds, J. reine angew. Math. 455 (1994) 183-220. MR 96e:53057

[7] J. L. Cathelineau. Deformations équivariantes d'espaces analytiques complexes compacts, Ann. Scient. Éc. Norm. Sup. $4^{e} 11$ (1978) 391-406. MR 80a:32018

[8] R. Godement. Théorie Des Faisceaux, Act. Sci. Ind. 1252, Hermann, Paris (1958). MR 21:1583

[9] P. Griffiths \& J. Harris. Principles of Algebraic Geometry, John Wiley \& Sons, New York, (1978). MR 80b:14001

[10] N. J. Hitchin, A. Karlhede, U. Lindström \& M. Roček. Hyperkähler metrics and supersymmetry, Comm. Math. Phys., 108 (1987) 535-589. MR 88g:53048

[11] E. Horikawa. On deformations of holomorphic maps I, J. Math. Soc. Japan, 25 (1973) 372396. II, J. Math. Soc. Japan, 26 (1974) 647-667. MR 50:5027

[12] S. Kobayashi. Differential Geometry of Complex Vector Bundles, Publications of the Math. Soc. Japan, Iwanami Shoten \& Princeton University Press, Princeton (1987). MR 89e:53100

[13] C. R. LeBrun. A rigidity theorem for quaternionic-Kähler manifolds, Proc. Amer. Math. Soc., 103 (1988) 1205-1208. MR 89h:53105

[14] C. R. LeBrun. Fano manifolds, contact structures and quaternionic geometry, International J. Math., 6 (1995) 419-437. MR 96c:53108

[15] P. Molino. Riemannian Foliations, Progress in Math., 73 Birkhäuser, Boston, (1988). MR 89b:53054

[16] H. Pedersen \& Y. S. Poon. Deformations of hypercomplex structures, J. reine angew. Math. 499 (1998), 81-99. CMP 98:15 
[17] S. M. Salamon. Quaternionic Kähler manifolds, Invent. Math. 67 (1982) 143-171. MR 83k:53054

[18] S. M. Salamon. Differential geometry of quaternionic manifolds, Ann. Scient. Éc. Norm. Sup., $4^{e} 19$ (1986) 31-55. MR 87m:53079

[19] A. F. Swann. Hyperkähler and quaternionic Kähler geometry, Math. Ann., 289 (1991) 421450. MR 92c:53030

[20] K. Yano \& M. Kon. Structures on Manifolds, World Scientific, Singapore, (1984). MR 86g:53001

Institut for Matematik og Datalogi, Odense Universitet, Campusvej 55, Odense M, DK-5230, DENMARK

E-mail address: henrik@imada.ou.dk

Department of Mathematics, University of California at Riverside, Riverside, CalIFORNIA 92521

E-mail address: ypoon@math.ucr.edu 